\title{
Teoría de Sistemas y Deconstrucción
}

\author{
SYSTEMS THEORY AND DECONSTRUCTION
}

Dr. Carlos Durán (cduran@uarcis.cl) Escuela de Ciencia Política, Universidad ARCIS (Santiago, Chile)

\begin{abstract}
The following article attempts to be a contribution to the reflection on the lines of continuity, proximity relations and possibles elements of differentiation on two theoretical fields of growing importance within the social sciences: theory of systems of Niklas Luhmann and the deconstruction of Jacques Derrida. Along with accounting for the extent of both theories for reflection on the foundations of the social sciences, this article tries to install some useful questions for self-observation of the epistemological foundations of this field of knowledge.
\end{abstract}

Key words: systems theory, deconstruction, knowledge, epistemology, social sciences

\section{Resumen}

El siguiente artículo reflexiona en torno a las líneas de continuidad, relaciones de proximidad y elementos de diferenciación posibles de detectar en dos campos teóricos de creciente protagonismo al interior de las ciencias sociales: la teoría de sistemas de Niklas Luhmann y la deconstrucción de Jacques Derrida. Junto con dar cuenta de los alcances de ambas teorías para la reflexión relativa a los fundamentos de las ciencias sociales, el artículo pretende instalar algunas interrogantes de utilidad para la auto-observación de los fundamentos epistemológicos de dicho campo del conocimiento.

Palabras clave: teoría de sistemas, deconstrucción, conocimiento, epistemología, ciencias sociales

\section{Introducción}

¿Qué pueden tener en común deconstrucción y teoría de sistemas?, ¿es posible establecer una analogía entre una disposición teórica a destacar la diseminación del sentido y un ejercicio de observación de unidades que, como los sistemas, constituyen justamente unidades de procesamiento de sentido?

En una primera mirada, la relación entre deconstrucción y teoría de sistemas se torna imposible. Nociones tales como las de "cierre operativo" o "autopoiesis" (solo por mencionar dos categorías recurrentemente presentes en la terminología del sociólogo Niklas Luhmann), por ejemplo, constituirían términos inconmensurables vis a vis nociones caras a la gramática derridiana tales como "diseminación", "iterabilidad" o "indecidibilidad".

Y sin embargo, una observación más atenta de los supuestos con los que opera la teoría de sistemas permite detectar una verosímil proximidad con la deconstrucción: la superación de la oposición sujeto/objeto, así como el reconocimiento de un ineludible "punto ciego" en toda observación y la centralidad de la "observación de observaciones" como mecanismo que hace posible la comprensión, los cuales son algunos aspectos que permitirían determinar una confluencia entre la disposición luhmanniana para con el "conocimiento" y algunas de las operaciones más típicamente deconstructivas, tales como la negación de la unidad del sentido, el reconocimiento de la indecidibilidad de las significaciones y su parasitaria instalación en marcos de saber sobre los cuales se opera deconstructivamente. 
La ambigua relación entre la teoría de sistemas de Luhmann y el deconstruccionismo de Derrida no es un tema nuevo, sino que por el contrario ha sido un tópico frecuentemente debatido. González, solo por referir una de las múltiples reflexiones sobre esta relación, sintetiza el vínculo entre deconstrucción y teoría de sistemas con las siguientes palabras: "Luhmann emerge con un curioso intento de desarrollar un pensamiento original vinculado a la cibernética y a la elaboración de una teoría científica de la sociedad, a la vez que hacía uso y resonaba con muchas de las ideas que se manejaban en los debates en torno al postmodernismo y la crisis de la modernidad, el estructuralismo y el postestructuralismo, como por ejemplo Jacques Derrida en las concepciones acerca del lenguaje o Michel Foucault en lo que tiene que ver con el poder" (2003:1).

No obstante lo arriba expuesto, esta eventual confluencia presenta algunos problemas necesarios de ser abordados. En primer lugar, y si la teoría de sistemas de Luhmann constituye una propuesta teórica fuertemente relacionada, en sus supuestos de base, con la deconstrucción: ¿significa esto que la deconstrucción puede devenir en una metodología a ser aplicada en el campo de las ciencias sociales?; ¿significaría, acaso, que la deconstrucción puede tener mucho en común con la comprensión que generan las observaciones de segundo orden?; ¿significa, en consecuencia, que la deconstrucción puede devenir en un constructo epistemológico, en una producción de conocimiento certero? Probablemente, una respuesta afirmativa podría conducir a aseverar que lo que llama a "escándalo" al momento de vincular teoría de sistemas y deconstrucción no sería otra cosa que una mera cuestión de "acentos". Una cuestión de acentos, digo, aludiendo a la posibilidad de que categorías tan aparentemente certeras como la de sistema no sean otra cosa que la cara reversa de la constitutiva indecidibilidad que hace posible, al mismo tiempo que imposible, el sentido, o que la concepción que propone Luhmann acerca del conocimiento se encuentre plenamente advertida de la naturaleza contingente de todo sentido.

En el presente trabajo me he propuesto indagar en algunos aspectos que permitirían instalar a la teoría de sistemas y la deconstrucción en un campo común. Para ello, me concentraré en el concepto de "observación de segundo orden", el cual constituye de acuerdo a la sociología de Luhmann el espacio privilegiado desde el cual "los sistemas comprenden a los sistemas" y, por consecuencia, desde donde puede ser pensada una teoría sociológica capaz de abandonar los presupuestos ontológicos sobre los cuales ésta se ha desarrollado hasta ahora.

En la primera parte del trabajo, me aproximaré a lo que se podría llamar un "acuerdo negativo" que vincula a la teoría de sistemas y la deconstrucción. Me refiero específicamente al acuerdo en torno a la crítica de la "epistemología tradicional" (Luhmann) y la "metafísica de la plena presencia" (Derrida); en la segunda parte, intentaré dar cuenta de algunos de los aspectos centrales de la teoría luhmanniana de sistemas, concentrándome en los supuestos que rondan a la noción de observación de segundo orden para, en la tercera parte, confrontarla con algunos de los ejes centrales de lo que podría ser entendida como una "práctica deconstructiva".

\section{La deconstrucción y la teoría de sistemas frente a la teoría del conocimiento}

Desde que -en el ya lejano siglo XIX- Dilthey estableció la célebre distinción entre "ciencias de la naturaleza" y "ciencias de la cultura", aquello que se constituye como objeto de las ciencias sociales se ha asumido como un "problema en sí mismo", un espacio reflexivo condicionado por la pérdida de la culpable ingenuidad de suponer que el mundo puede ser aprehendido por medio de una "estricta presentación de los hechos", ya como lo planteaba el historiador alemán Leopold Von Ranke. Si ello es así, ¿cómo será posible entonces dar con su significado, sus contenidos y sus pautas de existencia? Tal ha sido, en definitiva, la pregunta que ha marcado la deriva de toda una tradición de pensamiento que ha intentado resolver positivamente, desde un lugar alejado de las coordenadas positivistas, la compleja relación entre el sujeto que conoce y el sentido por conocer. Deriva que, 
por ejemplo, incluye a la fenomenología, la hermenéutica, el interaccionismo simbólico o la teoría de la acción comunicativa de Jurgen Habermas.

Ahora bien, y pese al carácter afirmativo con que se resuelve la pregunta acerca de la posibilidad del conocimiento, nos encontramos en un campo en el cual, se reconoce, éste no se obtiene de modo transparente y directo. Obsérvese en este sentido la siguiente lectura que Luhmann ofrece en alusión a la estrategia "idealista" de superación del problema de la relación entre conocimiento y realidad: "En la tradición de la teoría idealista del conocimiento se trataba de la pregunta por la identidad de la diferencia entre conocimiento y objeto real. La pregunta era: ¿Cómo puede el conocimiento aprehender un objeto que está situado fuera de él? O: ¿cómo puede el conocimiento afirmar que algo puede existir independientemente de él, cuando todo lo que se aprehende ya presupone conocimiento y no puede por consiguiente ser independiente y, al mismo tiempo, ser afirmado por el conocimiento? Mientras se trató de resolver el problema de forma teórica trascendental o dialéctica se trató siempre de este problema: ¿Cómo es posible el conocimiento, si no puede haber ninguna relación con la realidad independiente del conocimiento?" (1998:70).

El conocimiento, entonces, se vincula ya en esta tradición en una paradojal relación con "aquello que conoce", toda vez que se asume que el acto mismo del conocimiento forma parte de la realidad por conocer. ¿Cómo responder a este impasse? De acuerdo a Luhmann, la resolución a la paradoja de un conocimiento como contenido y continente se intentó generar, con el afán de mantener la dualidad entre sujeto y objeto, configurando la idea de un "mundo en común" posible de ser aprehendido por medio de la "introspección". Sigamos a Luhmann: "La teoría del sujeto del conocimiento (...) siempre trató de resolver el círculo de la paradoja mediante introspección, en el sentido de cómo los otros se comportaban frente al mundo. Pudo conceder que no hay ninguna entrada directa a la vivencia de otro sujeto; pero cuando menos uno podía volverse al fenómeno de la propia conciencia, con cuyos principios también los otros ordenan los objetos en el mundo. La teoría del sujeto debió de presuponer un mundo observable en común y con ello pidió pensar el desacoplamiento de cada uno de los sistemas cognoscentes como condición de posibilidad del conocimiento" (1998:72).

Concentrémonos brevemente en la tradición fenomenológica, la cual podemos entender como una de las que con más fuerza intentó "salvar" la referida dualidad sujeto-objeto como condición de posibilidad del conocimiento. La fenomenología, claro está, no se encuentra ajena a la sospecha respecto a la transparencia de la atribución y transmisión de sentido, por lo que se propone "ir más allá" de las distinciones y categorizaciones objetivistas basadas en la confianza en que el "yo del otro" se presenta como algo dado transparentemente a la interpretación. Sin embargo, este reconocimiento respecto a la opacidad del sentido que se transmite al otro no clausura la posibilidad de su aproximación por parte de un sujeto de la comprensión. Tal es el rol que juega por ejemplo, en la sociología de Schutz, la noción de "co-simultaneidad" o "co-presencia": "mis vivencias de ti están constituidas en simultaneidad o casi simultaneidad con tus vivencias. Solo debido a esto ocurre que, cuando miro hacia atrás, soy capaz de sincronizar mis vivencias pasadas de ti con tus vivencias pasadas" (1993:136).

Con la noción de co-presencia, así, la fenomenología de Schutz elude los equívocos a los que a su juicio conduce la concentración en los "indicadores objetivos" del sentido subjetivo de la acción. Para ello, el sujeto de la comprensión hace uso de un "contexto de experiencia" que acerca su propia vivencia a la vivencia significada del yo del otro. Como vemos, es precisamente el acto de la "auténtica comprensión del yo del otro" el que se constituye como manifestación de una distancia del sujeto de la comprensión respecto al yo del otro o, dicho en otros términos, como el índice total de aproximación posible frente a la ausencia que "se presenta". Puesto que no podemos conocer el yo del otro, y puesto que las "indicaciones" siempre son una objetivación equívoca del sentido 
subjetivo, sólo queda por consecuencia aprehender el sentido por medio de un acto de comprensión posibilitado por los contextos de significado, acto que sin embargo siempre es solo "aproximativo", y que opera en función de un advertido "como sí": el "como sí" de la plena presencia del otro.

¿Qué podemos concluir de esto? Para Luhmann, la urgencia fenomenológica por mantener la posibilidad de aprehensión de un sentido pleno y estable, aun advertidas las dificultades que ello acarrea, implica la presencia de una serie de supuestos frente a los cuales su propuesta teórica pretende constituirse como superación. Me refiero, concretamente, a los supuestos de una dualidad sujeto-objeto, de una dualidad realidad-conocimiento, y a la fuerte presencia, en última instancia, de una ontología que supone la "unidad de lo existente".

En el caso de la hermenéutica ocurre algo similar. El reconocimiento tanto de la "distancia histórica" (Gadamer) como de la "otredad", que se encuentran en el corazón de la aproximación hermenéutica al conocimiento y su particular concepción de la interpretación, convive problemáticamente con la búsqueda por dar con esa distancia y esa otredad. En otros términos: si la distancia con el otro (un otro diacrónico o sincrónico) está presente en toda mirada, dicha mirada debe ser capaz de, en algún punto, "domesticar" esa otredad, dialogar con ella, comprenderla, aprehenderla. En fin: conocerla. Esta situación paradojal es ejemplificada por Paul Ricoeur en la dicotomía entre Verdad y Método presente en la propuesta hermenéutica de Gadamer: “...por un lado...el distanciamiento alienante es la actitud a partir de la cual es posible la objetivación que rige en las ciencias del espíritu o ciencias humanas; pero este distanciamiento, que condiciona el estatuto científico de las ciencias, es al mismo tiempo lo que invalida la relación fundamental y primordial que nos hace pertenecer y participar de la realidad histórica que pretendemos erigir en objeto. De allí la alternativa subyacente en el título mismo de la obra de Gadamer, Verdad y Método: o bien practicamos la actitud metodológica, y así perdemos la densidad ontológica de la realidad estudiada, o bien practicamos la actitud de verdad, pero entonces debemos renunciar a la objetividad de las ciencias humanas" (2004:95).

Cosa no muy distinta ocurre con la así llamada "tradición de la sospecha" de la cual formarían parte campos tan diversos como el psicoanálisis y la sociología. Observemos el argumento de Luhmann en relación a esto: “El observador primero era llevado a la zona de lo inocuo, de la ingenuidad, o era tratado como alguien que, sin saberlo, escondía algo. El conocimiento mejor se coló mediante la interposición de una sospecha y esta generalización del principio de la sospecha posibilitó el que disciplinas enteras -desde el psicoanálisis hasta la sociología- se establecieran en el mundo con una competencia colateral con la que cada una sabe, o al menos presume que sabe, de qué situación se trata" (1998:110).

Sospecha como búsqueda del "fundamento oculto de las cosas", como búsqueda del sentido último de las cosas. Sospecha, a fin de cuentas, como búsqueda por aprehender la "unidad de lo múltiple". Tal es, análogamente, la objeción que señala Derrida en relación a una hermenéutica de la sospecha que, para el filósofo argelino, lejos de sentenciar el fin de la metafísica de la plena presencia, se instaló en su corazón mismo. En palabras de Ferraris: “La voluntad de desenmascarar -de echar luz más allá del velo de las apariencias, de llegar a lo propio que se esconde detrás de la metáfora- no es el acto final de la metafísica, el mediodía de los espíritus libres del que habla Nietzche; al contrario, es precisamente el acto inicial de cualquier metafísica. Por otro lado, la metafísica no lo es en tanto que ignora que la misma 'verdad' no es más que una antigua metáfora: lo es, más bien, porque, consciente del carácter metafórico de los propios enunciados, ha intentado, a lo largo de toda su 'historia', reducir lo metafórico a lo propio, a lo adecuado, a lo conceptualmente unívoco" (1990:346).

Tanto en Luhmann como en Derrida, en definitiva, opera la misma distancia frente a toda una tradición de la filosofía del conocimiento. En ambos, opera una igualmente activa búsqueda por superar la ontología de la unidad 
del ser o, como lo señala Derrida, una activa búsqueda por superar aquella forma de enfrentarse al mundo "que pretende descifrar, sueña con descifrar una verdad o un origen que se sustraigan al juego y al orden del signo, y que vive como un exilio la necesidad de la interpretación" (1989:400).

\section{La imposible condición de posibilidad del conocimiento}

Si los supuestos sobre los cuales ha operado la teoría del conocimiento han quedado desmantelados, y si por tanto el conocimiento entendido en última instancia como adecuación o aprehensión del sentido constituye una imposibilidad: ¿qué queda entonces?; ¿es posible continuar hablando de conocimiento y, aún más, de comprensión? La respuesta de Luhmann a esta última interrogante será afirmativa. Es más, conceptos en principio tan fuertes tales como los de "ciencia", "sociedad", "realidad" y "verdad" reaparecerán de manera protagónica en la propuesta del sociólogo alemán, aunque esta vez procesados de manera estrictamente opuesta, creo posible afirmar, a la forma en que fueron utilizados por la "tradición moderna".

¿Cómo será posible entonces seguir pensando en la posibilidad del conocimiento? La respuesta de Luhmann podemos comenzar a aprehenderla una vez que damos con el desplazamiento que pretende sostener en relación a lo propio del conocimiento: "El conocimiento es solo posible porque no puede ponerse en contacto con la realidad" (1998:70). Para entender el sentido de esta afirmación, es preciso situarla al interior de lo que ha de asumirse como un intento constructivista por asumir la autorreferencialidad del conocimiento, entendido esto ya no como un problema a superar sino que más bien como una afirmación "ontológica": "Existe un mundo externo, lo que provoca que el conocimiento como una operación autónoma pueda ser guiado; pero no tenemos ningún tipo de acceso privilegiado hacia él. El conocimiento no puede tener acceso a la realidad sino mediante conocimiento. Se trata, con otras palabras, de un proceso autorreferencial. El conocimiento sólo se puede conocer a sí mismo (...) el conocimiento tiene que ver con un mundo externo que permanece desconocido, y debido a ello debe aprender consecuentemente a ver que no puede ver lo que no puede ver" (Luhmann 1998:93).

Sobre la base de tal desplazamiento en la concepción del conocimiento ejercida por Luhmann es que se hace posible pensar a los "sistemas" como su unidad objetual básica, unidad sólo posible de ser pensada si partimos del reconocimiento de la imposibilidad del acceso a la realidad "tal cual es", condición de posibilidad para la superación definitiva tanto de los enfoques subjetivistas como objetivistas del conocimiento. En palabras del propio Luhmann: "Nosotros lo que proponemos es sustituir la diferencia sujeto/objeto, por la de sistema/entorno. Por un lado, esta distinción sigue perteneciendo a la posición clásica del problema en la medida en que toma su punto de partida de una diferencia, y en la medida en que permite que una parte vuelva a reentrar en la otra. Sobrepasa, por otra parte, la posición clásica del problema, porque revierte tanto la teoría del sujeto como la del objeto. Esta posición puede sustituir la pregunta por el desacoplamiento del sistema mediante cerradura, por la de la diferenciación de los sistemas, y puede sustituir la premisa de un mundo común, por una teoría de la observación de los sistemas que observan" (1998:72).

Lo arriba indicado implica dos cuestiones de suma relevancia que aquí solo enunciaré brevemente: en primer lugar, la noción de sistema no podría "representar" una referencia a una exterioridad cualquiera. No correspondería asumirlo, por lo tanto, como una "interioridad" en relación a una "exterioridad" supuesta, de lo cual se deduce que su constitución-reproducción no puede ser otra cosa que un proceso autopoiético; en segundo lugar, y puesto que todo sistema constituye en último término una "totalidad", algo debe quedar fuera para que el sistema se constituya como tal. Sin embargo, aquello que queda fuera debe ser pensado como el efecto mismo del sistema, es decir, como el resultado de una operación que se debe asumir como primaria en todo sistema: la distinción entre sistema y entorno. Y es esto, precisamente, lo que define el carácter autopoiético de todo sistema, en la medida en 
que es el resultado de operaciones de autoorganización a partir de una constante distinción sistema-entorno que el propio sistema realiza de manera recursiva. En sus palabras: "Como recursivo se designa aquel proceso que utiliza sus propios resultados como plataforma para las operaciones inmediatamente subsecuentes; por tanto, lo que se emprenderá estará codeterminado por las operaciones contiguamente anteriores" (Luhmann 1998:107).

El entorno, consecuentemente con lo arriba señalado, siempre ha de ser asumido como relativo a un sistema (correlato negativo del sistema), esto es, como un modo de azar definido como tal desde el punto de observación de un sistema. En palabras de Izuzquiza: "una porción de azar que el sistema reconoce como abierta ante sí y que es, por otra parte, condición de su propia existencia. No puede haber sistema sin un ámbito de posibilidades respecto a las que éste pueda ejercitar su selección. Y este ámbito no es más que el entorno" (1990:159).

Claro está, entonces, que lo que nombra la teoría de sistemas no es una unidad como tal, sino que más bien una operación -la diferencia- que contiene en sí misma una operación de conocimiento: "una teoría debe estar estructurada según la lógica de la diferencia. Debe ofrecer posibilidades para diferenciar, para establecer distinciones, más que para construir unidades. Una teoría será adecuada en tanto pueda tratar con diferencias, pueda crear nuevas diferencias y procesarlas de un modo siempre dinámico" (Izuzquiza 1990:44).

Realizado este sumario trayecto por la concepción de sistema ofrecida por Luhmann es que puedo retornar a la pregunta relativa a la posibilidad de un conocimiento que asume la imposibilidad de "aprehender" algo fuera de su propia operación. El conocimiento, desde esta perspectiva, se encuentra estrechamente ligado a la operación de observación que cada sistema hace respecto a sus propias operaciones, y sólo es posible de ser pensado como efecto del carácter autopoiético de las mismas: "El conocimiento se definirá mediante operaciones de observación y de descripción de las observaciones. Esto incluye observaciones de las observaciones y descripciones de las descripciones (...) siempre debe ser el observar y describir una operación autopoiética, por consiguiente una realización biológica o una conciencia actual o comunicación; de otra manera no se podría reproducir la cerradura y la diferencia del sistema cognoscente, puesto que la observación no se llevaría a cabo en el sistema mismo" (Luhmann 1998:74).

Todo conocimiento, por lo tanto, opera a partir de un doble ejercicio de distinción (por medio de lo cual se produce la diferencia entre sistema y entorno) e indicación (por medio de la cual se especifica lo propio del sistema) más allá del cual no existe una "realidad" esperando a ser aprehendida. Y es que, en definitiva, la operación propia del sistema (el conocimiento) es lo que constituye al mismo tiempo el ejercicio y el objeto del conocimiento: "Todas las distinciones e indicaciones son puras operaciones recursivas de un sistema (...) y estas operaciones no pueden salir del sistema, ni pueden traer algo fuera hacia adentro mediante, por así decirlo, una mano larga. Todas las adquisiciones, sobre todo aquellas que se designan como información, son resultado puramente interno. No hay ninguna información que pueda ser traída desde fuera hacia adentro, ya que precisamente la diferencia y el horizonte de posibilidades por las que la información puede llegar a ser una selección no existen en el entorno, sino que se trata de un constructo interno del sistema" (Luhmann 1998:102).

Concebido así, el conocimiento se asume como una operación que ya no se enfrenta al drama de su escisión con la realidad, en la medida en que el mundo, entendido como una "unidad" a ser aprehendida, deja de ser concebido como tal. En palabras de Luhmann: "El efecto de esta intervención de la teoría de sistemas puede ser descrito como una desontologización de la realidad. Esto no significa que se ignore la realidad (...) lo que se pone en tela de juicio es sólo la relevancia teórica para el conocimiento de una representación ontológica de la realidad” (1998:99). 
Tal como es posible deducir de lo expuesto hasta aquí, un sistema ha de ser asumido como una unidad que se constituye por medio de su propia observación. Las operaciones propias a este nivel serán denominadas como "observación de primer orden", entendida como una operación que produce al mismo tiempo que es efecto del propio sistema y sus contornos (diferencia sistema-entorno) creados por la misma operación de observación. Y dicha operación es, justamente, lo que debemos entender por conocimiento: "El conocimiento sólo es posible cuando y debido a que el sistema, en el plano de sus distinciones y descripciones, se cierra operativamente y de esa manera crea una diferencia con respecto a lo que se designa como entorno. La consideración de que el conocimiento sólo es alcanzable mediante el rompimiento que llevan a cabo las relaciones operativas con respecto al entorno, no quiere decir que el conocimiento no sea algo real. Lo único que afirma es que para las operaciones con las que un sistema cognoscente se diferencia, no puede haber nada que corresponda con el entorno; porque, si hubiera algo fuera que correspondiera, el sistema permanentemente se disolvería en el entorno y con ello el conocimiento sería imposible" (Luhmann 1998:89).

Pues bien, el límite de la observación de primer orden se encuentra signado precisamente por el hecho de que ésta se realiza al interior de los marcos mismos del sistema al cual pertenece. Es decir: el límite de la observación de primer orden es el propio sistema, con lo cual ésta no puede ni representar su entorno ni la propia distinción entre sistema/entorno sobre la cual se sostiene. Todo sistema, por lo tanto, opera a condición de un límite, un punto ciego, una autoobservación imposible. Y ello implica, por consecuencia, que todo sistema opera en torno a una paradoja constitutiva, consistente en su imposibilidad de conocer lo que conoce y lo que no conoce. Tal es, justamente, el dilema de la imposibilidad del conocimiento al cual he hecho referencia más arriba. Obsérvese en este sentido el siguiente argumento de González: "El conocimiento es necesario -de hecho imprescindible- para el sistema, porque su reproducción como sistema depende de su continuo y permanentemente exitoso acoplamiento a su entorno. Pero es imposible porque el sistema no tiene acceso al entorno, ya que todas sus operaciones se dan al interior y en virtud de la organización del propio sistema. $\mathrm{O}$, dicho de otro modo, las operaciones llevadas a cabo por el sistema como reacción a lo que percibe como estímulo del entorno, depende de su organización interna y no de las características del entorno. El sistema es ciego a su entorno, aunque debe acoplarse continuamente a él para seguir existiendo. El hecho de que exista y siga evolucionando es evidencia de que su relación es exitosa, es decir que conoce su entorno, a pesar de que no conoce lo que conoce" (2003:7).

Por otro lado, sin embargo, es este mismo desconocimiento del entorno lo que posibilita el conocimiento, toda vez que produce el cierre operativo que permanentemente realiza cada sistema en relación a su entorno y sobre sí mismo. Y es que, en definitiva, "el punto ciego de la distinción que utiliza cada observación es, al mismo tiempo, su garantía de mundo" (Luhmann 1998:103).

¿Puede por consecuencia el sistema-observador "observar" la diferencia que lo constituye? Evidentemente no. Y para ello están las observaciones de segundo orden, entendidas como operaciones de observación (que evidentemente provienen de un sistema que observa a otro sistema y que, eventualmente, puede ser observado en sus operaciones por otro sistema) que tienen como función central el enfrentarse a lo que el observador de primer orden no puede ver, es decir, las distinciones sobre las cuales éste opera, llamadas también "estructuras latentes". La observación de segundo orden, así, asume como objeto privilegiado de comprensión a las formas que asumen, en un sistema, las paradojas que lo constituyen, dando cuenta al mismo tiempo de la manera en que éstas paradojas son sorteadas por el propio sistema para asegurar su operatividad. De lo que se trata, entonces, es de comprender "cómo otro observador invisibiliza sus propias paradojas" (Luhmann 1998:112). 
En la observación de segundo orden, en definitiva, "se observa (...) la distinción con la que el primer observador observa, y cómo él en la realización de la observación de esta distinción no puede distinguirla, y por tanto se observa lo que para él es inconsciente o permanece incomunicable. En jerga que es específica de la sociología se puede decir: el observar se dirige ahora a las estructuras y funciones latentes del observador observado" (Luhmann 1998:109).

Observación de segundo orden, por lo tanto, entendida como visibilización de los ejercicios binarios (siempre paradójicos) sobre los cuales un sistema se constituye. Visibilización que, no obstante, y lejos de interrumpir la posibilidad de la comprensión, constituyen su razón de ser: "El paso de la pregunta por la relación a la unidad, se sustituye por la pregunta de un uso operativo de la diferencia, y allí se puede reconocer que con ello el círculo tautológico y las paradojas no se pueden soslayar sino que forman parte del juego" (Luhmann 1998:95).

\section{Conocimiento, deconstrucción y paradoja}

He concluido el apartado anterior dando cuenta de la relevancia que la visibilización de las codificaciones binarias propias a todo sistema tiene para la noción de observación de segundo orden desarrollada por Luhmann. He terminado con esto, atendiendo a la relevancia análoga que, para la deconstrucción, manifiesta el ejercicio de visibilización de los códigos binarios presentes en las operaciones de construcción de significado. Ahora bien, ¿significa esta concentración en las codificaciones binarias propia de la deconstrucción y la teoría de sistemas un argumento como para determinar la existencia de una relación de contigüidad entre ambos paradigmas?

Tal como se ha planteado ampliamente desde distintos lugares (Asensi, Rojo, Culler), es en el campo de la teoría literaria que la deconstrucción ha encontrado un lugar fértil en el cual expresarse. Y ello no resulta extraño, debido a que en primer lugar su objeto se presenta como exclusivamente escritural y, en segundo lugar, debido a que la teoría literaria siempre ha sido entendida como un lugar que se vincula conflictivamente con el campo de la ciencia, en una paradojal relación de frontera. Pero ahora, ¿puede la deconstrucción operar en el ámbito de las ciencias sociales en general? Desde el canon disciplinar, la respuesta debiera ser rotundamente negativa. Y los argumentos para ello serían básicamente dos. En primer lugar, por cuanto las ciencias sociales habitan una forma particular de lenguaje, el lenguaje lógico-argumentativo, que no requiere de la inserción de una exterioridad que explicite sus paradojas. Ello, en la medida en que las paradojas, las contradicciones y la indecidibilidad no formarían parte de dicha forma del lenguaje.

En segundo lugar, y a diferencia de la teoría literaria, las ciencias sociales operarían en función de un "exterior" que impediría asumirlas como un "universo en sí misma". Ese exterior, constituido por la "realidad social", sería el que en última instancia sirve de árbitro para distinguir, de acuerdo a los cánones generales de la ciencia, entre una producción textual adecuada y una producción textual inadecuada.

De acuerdo a estos criterios, por consecuencia, la generación de una lectura deconstructiva en el ámbito de la textualidad de las ciencias sociales no sería otra cosa que un ejercicio infértil, toda vez que tanto las reglas del lenguaje lógico-argumental como la existencia de una "realidad externa" a la propia producción textual dotarían a éstas de los instrumentos que hacen posible su "auto-observación" y "evaluación".

¿Cómo disponer a la deconstrucción frente a esta distancia pretendida por las ciencias sociales? En su texto La estructura, el signo y el juego en el discurso de las ciencias humanas Derrida destacaba, con el conocido ejemplo del efecto desestabilizador que en la oposición estructuralista entre naturaleza y cultura generaba el factum de la prohibición del incesto, la precariedad de las oposiciones binarias propias del saber moderno (Derrida 1989:390). 
Mostraba así una posible forma de ingresar la deconstrucción al campo del saber moderno: explicitar sus paradojas por medio de la visibilización de las oposiciones binarias jerárquicas a través de las cuales dicho saber estabiliza el sentido de su textualidad. Para Culler, la visibilización del carácter metafísico de las oposiciones binarias reseñadas constituyen quizás la operación por excelencia de todo ejercicio deconstructivo: “La deconstrucción no aclara los textos en el sentido tradicional de intentar captar un contenido o tema unitario; investiga el funcionamiento de las oposiciones metafísicas en sus argumentos y los modos en que las figuras y las relaciones textuales... producen una lógica doble y aporética" (1992:99).

¿En qué consiste la paradoja? Antes que nada, la paradoja se dirige hacia el lugar mismo de los géneros, de las distinciones entre lenguajes, entre campos de saber, entre el saber y aquello que se sabe. Para lo que nos ocupa, se dirige hacia las dos oposiciones que sustentan el lenguaje de las ciencias sociales: la oposición entre lenguaje argumentativo y lenguaje literario, por una parte, y la oposición entre exterior e interior.

Con respecto a la primera oposición (entre lenguaje argumental y lenguaje literario), existe una multiplicidad de lugares desde los cuales dicha oposición ha sido desestabilizada. Richard Rorty, por ejemplo, plantea que la distancia entre el lenguaje argumental y el lenguaje metafórico es equivalente a la distancia entre la "metáfora viva" y la "metáfora muerta. Es decir, corresponde meramente a una cuestión si se quiere "temporal" de distinción entre el lugar de lo instituido y el movimiento instituyente. Desde el campo de la teoría literaria, por su parte, se ha desarrollado ampliamente un ejercicio de desestabilización de la oposición referida, atendiendo a la puesta en cuestión de los criterios de frontera en los cuales ésta se funda. Es así como las especificidades que desde uno $u$ otro polo apelan a criterios tales como la "ficcionalidad", la "retoricidad", la "universalidad", la "lógica", los "criterios de verdad", se han diluido en la explicitación de una distancia "indecidible" entre ambos lenguajes (1).

La segunda oposición reseñada -la oposición entre interioridad y exterioridad- se instala como estrategia para la fundación de los criterios de objetividad que definen a toda ciencia en general, y a las ciencias sociales en particular. Y sin embargo, la distinción interior-exterior constituye un criterio de delimitación igualmente inestable, en la medida en que el "afuera", junto con ser constituido desde el "interior", opera como condición de posibilidad de este último. Por lo demás, y si todo acto de "nominación" de aquel exterior definido como objeto ha de ser entendido tanto en su dimensión performativa como en su carácter de reducción inevitable de aquello que se nombra, la "ilusión de verdad" de las categorías que conforman el léxico de las ciencias sociales se vuelve a lo menos objetable, tan objetable como la confianza de una representación del mundo que funda una verdad sobre la base de su reducción categorial.

Como podemos percibir, la puesta en cuestión de las oposiciones entre "lenguaje argumentativo" y "lenguaje figurativo", así como la desestabilización de la oposición entre exterior e interior sobre la cual se fundan las ciencias sociales constituye un ejercicio que, pensado en términos de teoría de sistemas, bien pudiera ser definida como 1) una observación de los "puntos ciegos" presentes en toda observación sociológica que solo puede ser observada por un "observador de segundo orden" y 2) una constatación ontológica de la inexistencia de un objeto pleno y estable esperando ser aprehendido por un sistema de saber.

Pero no es solo el ejercicio análogo de visibilización de las paradojas lo que acerca deconstrucción y teoría de sistemas. Además, y al igual que la teoría de sistemas, la deconstrucción opera sólo a condición de situarse en un "contexto", prevenida contra todo posicionamiento en una atalaya capaz de distinguir lo verdadero de lo falso, lo eficiente de lo ineficiente. Sin contexto, no hay deconstrucción, de la misma manera en que sin sistema no hay "comprensión de los sistemas". Junto a ello, tanto para la deconstrucción como para la teoría de sistemas, su "objeto privilegiado" (la escritura y los sistemas, respectivamente), son objetos "sin sujeto". De la misma manera, 
escritura y sistemas alcanzan un carácter autorreferencial. Y ello implica que, por una parte, el sistema no media entre hombres y mundo, ni la escritura entre sujeto y sujeto. La escritura no es un medio, sino que un contenido en sí mismo, de la misma manera en que el conocimiento de los sistemas implica una operación que al mismo tiempo constituye el propio objeto de conocimiento.

\section{Conclusión}

En este trabajo, me he propuesto dar cuenta de algunas de las relaciones de proximidad posibles de determinar en dos expresiones teóricas a primera vista inconmensurables entre sí: la teoría de sistemas y la deconstrucción.

¿Significa lo expuesto en este texto que deconstrucción y teoría de sistemas bien pueden ser fundidos en un mismo paradigma? Para Teubner, el análogo tratamiento de las paradojas en la deconstrucción y la teoría de sistemas debe aguardar, antes del veredicto definido acerca de la proximidad entre ambas estrategias, el momento en que los "estrechos senderos" se bifurcan. La visibilización de las paradojas puesta como momento central de la operación deconstructiva opera como un mecanismo de diseminación, de multiplicación de sentidos cuyo destino es la desestabilización, entendida como afirmación ontológica de la diferencia. En el caso de la "observación de observaciones" propia de la teoría de sistemas, por el contrario, la observación de segundo orden opera en términos de "reducción de complejidad", lo que en otros términos pudiera entenderse como producciónaprehensión del sentido de lo observado, más allá de las paradojas por medio de las cuales dicho sentido se expresa. Mientras la deconstrucción operaría en la dirección de afirmar la indecidibilidad y el límite de todo saber, la teoría de sistemas operaría en términos de dar con las operaciones de clausura con que todo sistema asegura sus condiciones de existencia. De esta forma es que, en la teoría de sistemas, "la paradoja de la distinción que no se puede distinguir a sí misma no será resuelta, sino que será traída a una forma en la que se haga útil su procesamiento. Expresado de otra manera: el problema del último fundamento será estratégicamente evadido" (Gripp-Hagelstange 2004:39).

En definitiva, mientras que la deconstrucción "sirve, ante todo, como un ejercicio ontológico, como indicación de la inconmensurabilidad del observador respecto al objeto de la comprensión" (Ferraris 1990:354), la teoría de sistemas operaría con la pretensión de avanzar un paso más, asumiendo la inconmensurabilidad entre el observador y lo observado para, a partir de allí, generar conocimiento, es decir, "comprender a los sistemas". Y es precisamente en este momento en que, a mi juicio, comienza a visibilizarse de manera más prístina la distancia entre deconstrucción y teoría de sistemas. Lo que en un primer momento aparecía como una mera "afirmación metafórica" -el carácter científico de la teoría de sistemas- reaparece ahora bajo su forma plena: una metodología para el "conocimiento" de la operatoria de los sistemas.

Mientras la teoría de sistemas opera a partir de la visibilización de las paradojas con el objetivo de "comprender a los sistemas", el ejercicio deconstructivo, irreductible a toda traducción metodológica, se dirige por la ruta de la afirmación de la diferencia, de la diseminación. Si como señala Teubner, deconstrucción y teoría de sistemas coinciden en la afirmación de la paradojal relación entre "derecho, fuerza y justicia", las distancias entre ambos paradigmas se visibilizarán en el momento en que, mientras la teoría de sistemas daría cuenta del "cierre operativo" que resuelve la paradoja del Derecho, la deconstrucción afirmaría la a-científica, a-metodológica e "inoperante" posibilidad de la justicia. 


\section{Notas}

(1) Para una referencia a los contenidos específicos de la distinción-indistinción entre el lenguaje argumentativo y el lenguaje poético, ver Rojo (Diez Tesis sobre la Crítica).

(2) Las similitudes en el tratamiento de los "códigos binarios" con que opera la deconstrucción y la teoría de sistemas de Luhmann puede verse desarrollada en Teubner (Economics of Gift-Positivity of Justice. The Mutual Paranoia of Jacques Derrida and Niklas Luhmann. Theory Culture \& Society).

\section{Bibliografía}

Culler, J. 1992. Sobre la deconstrucción. Madrid: Cátedra.

Derrida, J. 1989. La escritura y la diferencia. Barcelona: Anthropos.

Ferraris, M. 1990. Jacques Derrida. Deconstrucción y ciencias del espíritu. En: M. Asensi (comp.). Teoría literaria y deconstrucción. Madrid: Arco / Libros, pp. 339-396.

González, E. 2003. Niklas Luhmann y la paradoja del conocimiento: algunas reflexiones acerca de la ciencia como sistema social autopoiético de comunicación. Red Cibernética de Estudios Intersdisciplinarios 1: 1-11

Gripp-Hagelstange, H. 2004. Niklas Luhmann o: ¿En qué consiste el principio teórico sustentado en la diferencia? En: J. Torres. Luhmann: la política como sistema. México: FCE, pp. 27-50.

Izuzquiza, I. 1990. La sociedad sin hombres. Niklas Luhmann o la teoría como escándalo. Barcelona: Anthropos.

Luhmann, N. 1998. Teoría de los sistemas sociales II (artículos). Barcelona: Anthropos.

Ricoeur, P. 2004. Del texto a la acción. Ensayos de hermenéutica II. México: FCE.

Schutz, A. 1993. La construcción significativa del mundo social. Barcelona: Paidós.

Recibido el 1 Oct 2012

Aceptado el 12 Ene 2013 